\title{
Different common fixed point theorems of integral type for pairs of subcompatible mappings
}

\author{
HAKIMA BOUHADJERA*
}

\begin{abstract}
In this paper, a general common fixed point theorem for two pairs of subcompatible mappings satisfying integral type implicit relations is obtained in a metric space. Our result improves several results especially the result of Pathak et al. [6]. Also, another common fixed point theorem of Greguš type for four mappings satisfying a contractive condition of integral type in a metric space using the concept of subcompatibility is established which generalizes the result of Djoudi and Aliouche [1] and others. Again a third common fixed point theorem for two pairs of near-contractive subcompatible mappings is given which enlarges the result of Mbarki [5] and references therein.
\end{abstract}

\section{INTRODUCTION}

Let $(\mathcal{X}, d)$ be a metric space and let $f, g$ be two mappings from $\mathcal{X}$ into itself. $f$ and $g$ commute if $f g x=g f x$ for all $x \in \mathcal{X}$.

This commutativity was weakened in 1982 by Sessa [7] with the notion of weakly commuting mappings. $f$ and $g$ above are weakly commuting if $d(f g x, g f x) \leq d(g x, f x)$ for all $x$ in $\mathcal{X}$.

Later on, Jungck [3] enlarged the class of commuting and weakly commuting mappings by compatible mappings which asserts that the above mappings $f$ and $g$ are compatible if $\lim _{n \rightarrow \infty} d\left(f g x_{n}, g f x_{n}\right)=0$ whenever $\left\{x_{n}\right\}$ is a sequence in $\mathcal{X}$ such that $\lim _{n \rightarrow \infty} f x_{n}=\lim _{n \rightarrow \infty} g x_{n}=t$ for some $t \in \mathcal{X}$.

This concept was further improved by Jungck [4] with the notion of weakly compatible mappings. $f$ and $g$ are weakly compatible if $f t=g t$ for some $t \in \mathcal{X}$ implies that $f g t=g f t$.

Recently in 2007, Pathak et al. [6] stated and proved a general common fixed point theorem of integral type for two pairs of weakly compatible mappings satisfying integral type implicit relations in a symmetric space.

2010 Mathematics Subject Classification. Primary: 47H10, 37C25, 54H25, 55M20.

Key words and phrases. Weakly compatible mappings, subcompatible mappings, implicit relations, common fixed point theorems, contractive and near-contractive conditions, Greguš type, metric space.

Full paper. Received 16 July 2018, revised 8 August 2018, accepted 28 August 2018, available online 15 December 2018. 
Our aim here is to improve and extend the result of [6] by using the new concept of mappings called subcompatibility which enlarges the concept of weakly compatible mappings.

We introduce the notion of subcompatible mappings as follows: Let $f$ and $g$ be two self-mappings of a metric space $(\mathcal{X}, d)$. $f$ and $g$ are subcompatible if and only if there exists a sequence $\left\{x_{n}\right\}$ in $\mathcal{X}$ such that $\lim _{n \rightarrow \infty} f x_{n}=\lim _{n \rightarrow \infty} g x_{n}=t$ for some $t \in \mathcal{X}$ and $\lim _{n \rightarrow \infty} d\left(f g x_{n}, g f x_{n}\right)=0$.

It is clear to see that weakly compatible mappings are subcompatible, however the implication is not reversible.

Example 1.1. Let $\mathcal{X}=[0, \infty)$ with the usual metric $d$. Define $f, g: \mathcal{X} \rightarrow \mathcal{X}$ as follows

$$
f x=x^{2} \text { and } g x= \begin{cases}x+12, & \text { if } x \in[0,16] \cup(25, \infty), \\ x+240, & \text { if } x \in(16,25] .\end{cases}
$$

Let $\left\{x_{n}\right\}$ be a sequence in $\mathcal{X}$ defined by $x_{n}=4+\frac{1}{n}$ for $n \in \mathbb{N}^{*}=\{1,2, \ldots\}$. Then, we have

$$
\lim _{n \rightarrow \infty} f x_{n}=\lim _{n \rightarrow \infty} x_{n}^{2}=16=\lim _{n \rightarrow \infty} g x_{n}=\lim _{n \rightarrow \infty}\left(x_{n}+12\right)
$$

and

$$
\begin{aligned}
& f g x_{n}=f\left(x_{n}+12\right)=\left(x_{n}+12\right)^{2} \rightarrow 256 \text { as } n \rightarrow \infty, \\
& g f x_{n}=g\left(x_{n}^{2}\right)=x_{n}^{2}+240 \rightarrow 256 \text { as } n \rightarrow \infty .
\end{aligned}
$$

Therefore, $\lim _{n \rightarrow \infty} d\left(f g x_{n}, g f x_{n}\right)=0$. Hence, $f$ and $g$ are subcompatible mappings.

On the other hand, we have $f x=g x$ if and only if $x=4$ but

$$
f g(4)=f(16)=256 \neq 28=g f(4)=g(16) .
$$

Thus, $f$ and $g$ are not weakly compatible.

For our first main result we need the following implicit relations.

\section{IMPLICIT RELATIONS}

Let $\mathbb{R}_{+}$be the set of all nonnegative real numbers, $\Psi$ be the family of all $\psi: \mathbb{R}_{+} \rightarrow \mathbb{R}$ Lebesgue-integrable and summable mappings and $\Phi$ be the set of all real continuous functions $\varphi: \mathbb{R}_{+}^{6} \rightarrow \mathbb{R}$ satisfying the following conditions:

$\left(\varphi_{1}\right)$ for all $u, v \geq 0$, if

$$
\begin{aligned}
& \left(\varphi_{a}\right) \int_{0}^{\varphi(u, v, v, u, 0, u+v)} \psi(t) \mathrm{d} t \leq 0 \text { or } \\
& \left(\varphi_{b}\right) \int_{0}^{\varphi(u, v, u, v, u+v, 0)} \psi(t) \mathrm{d} t \leq 0, \\
& \text { we have } u \leq v,
\end{aligned}
$$


$\left(\varphi_{2}\right) \int_{0}^{\varphi(u, u, 0,0, u, u)} \psi(t) \mathrm{d} t>0$, for $u>0$.

Example 2.1. Let $\varphi\left(t_{1}, t_{2}, t_{3}, t_{4}, t_{5}, t_{6}\right)=t_{1}-k \max \left\{t_{2}, t_{3}, t_{4}, \frac{t_{5}+t_{6}}{2}\right\}$, where $k \in(0,1)$ and $\psi(t)=t$. Then $\varphi$ is continuous and $\psi$ is a Lebesgue-integrable mapping which is summable. We have

$\left(\varphi_{1}\right)$ Let $u>0$ and $v \geq 0$. If $u>v$ then

$$
\begin{aligned}
\varphi(u, v, v, u, 0, u+v) & =\varphi(u, v, u, v, u+v, 0) \\
& =u-k \max \left\{u, v, \frac{u+v}{2}\right\} \\
& =u(1-k),
\end{aligned}
$$

then

$$
\int_{0}^{u(1-k)} t \mathrm{~d} t=\frac{1}{2} u^{2}(1-k)^{2} \leq 0
$$

impossible, hence $u \leq v$. If $u=0$, then $u \leq v$.

$\left(\varphi_{2}\right) \varphi(u, u, 0,0, u, u)=u(1-k)$, so

$$
\int_{0}^{u(1-k)} t \mathrm{~d} t=\frac{1}{2} u^{2}(1-k)^{2}>0
$$

for $u>0$.

Example 2.2. $\varphi\left(t_{1}, t_{2}, t_{3}, t_{4}, t_{5}, t_{6}\right)=\left(1+\alpha t_{2}\right) t_{1}-\alpha \max \left\{t_{3} t_{4}, t_{5} t_{6}\right\}$ $-\beta \max \left\{t_{2}, t_{3}, t_{4}, \frac{1}{2}\left(t_{5}+t_{6}\right)\right\}$, where $\alpha \geq 0$ and $0<\beta<1$ and $\psi(t)=1$.

$\left(\varphi_{1}\right)$ Let $u>0$ and $v \geq 0$. Suppose that $u>v$, then

$$
\begin{aligned}
& \varphi(u, v, v, u, 0, u+v)=\varphi(u, v, u, v, u+v, 0) \\
= & (1+\alpha v) u-\alpha \max \{u v, 0\}-\beta \max \left\{v, u, \frac{u+v}{2}\right\} \\
= & u(1-\beta),
\end{aligned}
$$

then

$$
\int_{0}^{u(1-\beta)} \mathrm{d} t=u(1-\beta) \leq 0
$$

which is impossible. Thus, $u \leq v$. If $u=0$, then $u \leq v$.

$\left(\varphi_{2}\right) \varphi(u, u, 0,0, u, u)=u(1-\beta)$, then

$$
\int_{0}^{u(1-\beta)} \mathrm{d} t=u(1-\beta)>0, \quad \text { for all } u>0 .
$$

Now, we state and prove our main results. We begin by the first one. 


\section{MAIN RESUlts}

Theorem 3.1. Let $f, g, h$ and $k$ be four mappings of a metric space $(\mathcal{X}, d)$ into itself such that

$$
\int_{0}^{\varphi(d(f x, g y), d(h x, k y), d(f x, h x), d(g y, k y), d(k y, f x), d(h x, g y))} \psi(t) \mathrm{d} t \leq 0,
$$

for all $x, y$ in $\mathcal{X}$, where $\varphi \in \Phi$ and $\psi \in \Psi$. Suppose that $(f, h)$ and $(g, k)$ are subcompatible and $h$ and $k$ are continuous, then, $f, g, h$ and $k$ have a unique common fixed point.

Proof. Since the pairs $(f, h)$ and $(g, k)$ are subcompatible, then, there exist two sequences $\left\{x_{n}\right\}$ and $\left\{y_{n}\right\}$ in $\mathcal{X}$ such that $\lim _{n \rightarrow \infty} f x_{n}=\lim _{n \rightarrow \infty} h x_{n}=t$ for some $t \in \mathcal{X}$ and $\lim _{n \rightarrow \infty} d\left(f h x_{n}, h f x_{n}\right)=0 ; \lim _{n \rightarrow \infty} g y_{n}=\lim _{n \rightarrow \infty} k y_{n}=z$ for some $z \in \mathcal{X}$ and $\lim _{n \rightarrow \infty} d\left(g k y_{n}, k g y_{n}\right)=0$.

First we prove that $z=t$. Indeed, by inequality (1) we get

$$
\int_{0}^{\varphi\left(d\left(f x_{n}, g y_{n}\right), d\left(h x_{n}, k y_{n}\right), d\left(f x_{n}, h x_{n}\right), d\left(g y_{n}, k y_{n}\right), d\left(k y_{n}, f x_{n}\right), d\left(h x_{n}, g y_{n}\right)\right)} \psi(t) \mathrm{d} t \leq 0 .
$$

Since $\varphi$ is continuous, we obtain at infinity

$$
\int_{0}^{\varphi(d(t, z), d(t, z), 0,0, d(z, t), d(t, z))} \psi(t) \mathrm{d} t \leq 0,
$$

which contradicts $\left(\varphi_{2}\right)$ if $d(t, z)>0$. Then, $z=t$.

Since $h$ is continuous, then $h^{2} x_{n} \rightarrow h t, h f x_{n} \rightarrow h t$. Also we have

$$
d\left(f h x_{n}, h t\right) \leq d\left(f h x_{n}, h f x_{n}\right)+d\left(h f x_{n}, h t\right) .
$$

Since $f$ and $h$ are subcompatible, taking the limit as $n \rightarrow \infty$ in the above inequality we have $\lim _{n \rightarrow \infty} f h x_{n}=h t$. The use of condition (1) gives

$$
\int_{0}^{\varphi\left(d\left(f h x_{n}, g y_{n}\right), d\left(h^{2} x_{n}, k y_{n}\right), d\left(f h x_{n}, h^{2} x_{n}\right), d\left(g y_{n}, k y_{n}\right), d\left(k y_{n}, f h x_{n}\right), d\left(h^{2} x_{n}, g y_{n}\right)\right)} \psi(t) \mathrm{d} t \leq 0 .
$$

At infinity we obtain

$$
\int_{0}^{\varphi(d(t, z), d(t, z), 0,0, d(z, t), d(t, z))} \psi(t) \mathrm{d} t \leq 0
$$

which contradicts $\left(\varphi_{2}\right)$. Hence $h t=t$.

Again using (1) we get

$$
\int_{0}^{\varphi\left(d\left(f t, g y_{n}\right), d\left(h t, k y_{n}\right), d(f t, h t), d\left(g y_{n}, k y_{n}\right), d\left(k y_{n}, f t\right), d\left(h t, g y_{n}\right)\right)} \psi(t) \mathrm{d} t \leq 0 .
$$

Taking the limit as $n \rightarrow \infty$, we get

$$
\int_{0}^{\varphi(d(f t, t), 0, d(f t, t), 0, d(t, f t), 0)} \psi(t) \mathrm{d} t \leq 0,
$$


which implies $d(f t, t)=0$ by using condition $\left(\varphi_{b}\right)$. Thus, $f t=t$.

Now, since $k$ is continuous we have $\lim _{n \rightarrow \infty} k^{2} y_{n}=\lim _{n \rightarrow \infty} k g y_{n}=k t$. Also we have

$$
d\left(g k y_{n}, k t\right) \leq d\left(g k y_{n}, k g y_{n}\right)+d\left(k g y_{n}, k t\right) .
$$

Since the pair $(g, k)$ is subcompatible we obtain at infinity $\lim _{n \rightarrow \infty} g k y_{n}=k t$. Using condition (1) we have

$$
\int_{0}^{\varphi\left(d\left(f t, g k y_{n}\right), d\left(h t, k^{2} y_{n}\right), d(f t, h t), d\left(g k y_{n}, k^{2} y_{n}\right), d\left(k^{2} y_{n}, f t\right), d\left(h t, g k y_{n}\right)\right)} \psi(t) \mathrm{d} t \leq 0 .
$$

When $n$ tends to infinity, we get

$$
\int_{0}^{\varphi(d(t, k t), d(t, k t), 0,0, d(k t, t), d(t, k t))} \psi(t) \mathrm{d} t \leq 0,
$$

which contradicts $\left(\varphi_{2}\right)$ when $d(t, k t)>0$. Hence, $k t=t$.

If $g t \neq t$, using inequality (1) we have

$$
\int_{0}^{\varphi(d(f t, g t), d(h t, k t), d(f t, h t), d(g t, k t), d(k t, f t), d(h t, g t))} \psi(t) \mathrm{d} t \leq 0,
$$

i.e.,

$$
\int_{0}^{\varphi(d(t, g t), 0,0, d(g t, t), 0, d(t, g t))} \psi(t) \mathrm{d} t \leq 0,
$$

which implies $d(t, g t)=0$ by using condition $\left(\varphi_{a}\right)$. Thus, $g t=t$.

For the uniqueness of common fixed point $t$, let $z \neq t$ be another common fixed point of $f, g, h$ and $k$. Then using (1) we obtain

$$
\int_{0}^{\varphi(d(f t, g z), d(h t, k z), d(f t, h t), d(g z, k z), d(k z, f t), d(h t, g z))} \psi(t) \mathrm{d} t \leq 0,
$$

that is,

$$
\int_{0}^{\varphi(d(t, z), d(t, z), 0,0, d(z, t), d(t, z))} \psi(t) \mathrm{d} t \leq 0,
$$

which is a contradiction of $\left(\varphi_{2}\right)$. Therefore $z=t$.

Corollary 3.1. Let $(\mathcal{X}, d)$ be a metric space and let $f$ and $h$ be two mappings from $\mathcal{X}$ into itself satisfying the condition

$$
\int_{0}^{\varphi(d(f x, f y), d(h x, h y), d(f x, h x), d(f y, h y), d(h y, f x), d(h x, f y))} \psi(t) \mathrm{d} t \leq 0,
$$

for all $x, y$ in $\mathcal{X}$, where $\varphi \in \Phi$ and $\psi \in \Psi$. If $h$ is continuous and the pair $(f, h)$ is subcompatible, then, $f$ and $h$ have a unique common fixed point.

Corollary 3.2. Let $(\mathcal{X}, d)$ be a metric space and let $f, g$ and $h$ be three self-mappings of $\mathcal{X}$ such that

(i) $h$ is continuous,

(ii) the pairs $(f, h)$ and $(g, h)$ are subcompatible and 
(iii) the inequality

$$
\int_{0}^{\varphi(d(f x, g y), d(h x, h y), d(f x, h x), d(g y, h y), d(h y, f x), d(h x, g y))} \psi(t) \mathrm{d} t \leq 0,
$$

holds for all $x, y$ in $\mathcal{X}$, where $\varphi \in \Phi$ and $\psi \in \Psi$, then, $f, g$ and $h$ have a unique common fixed point.

Now, we give a generalization of Theorem 3.1.

Theorem 3.2. Let $h, k$ and $\left\{f_{n}\right\}_{n \in \mathbb{N}^{*}}$ be mappings from a metric space $(\mathcal{X}, d)$ into itself such that

(i) the pairs $\left(f_{n}, h\right)$ and $\left(f_{n+1}, k\right)$ are subcompatible,

(ii) the inequality

$$
\int_{0}^{\left.\varphi\left(d\left(f_{n} x, f_{n+1} y\right), d(h x, k y), d\left(f_{n} x, h x\right), d\left(f_{n+1} y, k y\right), d\left(k y, f_{n} x\right), d\left(h x, f_{n+1} y\right)\right)\right)} \psi(t) \mathrm{d} t \leq 0
$$

holds for all $x, y$ in $\mathcal{X}$, each $n \in \mathbb{N}^{*}, \varphi \in \Phi$ and $\psi \in \Psi$. If $h$ and $k$ are continuous, then, $h, k$ and $\left\{f_{n}\right\}_{n \in \mathbb{N}^{*}}$ have a unique common fixed point.

Now, let $\mathcal{F}$ be the family of mappings $F: \mathbb{R}_{+} \rightarrow \mathbb{R}_{+}$such that each $F$ is upper semi-continuous and $F(t)<t$ for all $t>0$ and let $\Omega$ be the family of $\omega: \mathbb{R}_{+} \rightarrow \mathbb{R}_{+}$such that every $\omega$ is a Lebesgue-integrable mapping which is summable and $\int_{0}^{\epsilon} \omega(t) \mathrm{d} t>0$ for each $\epsilon>0$.

In their paper [1], Djoudi and Aliouche proved a common fixed point theorem of Greguš type for four mappings satisfying a contractive condition of integral type in a metric space using the concept of weak compatibility.

Our objective here is to improve, extend and generalize the result of [1] by using the notion of subcompatibility.

Theorem 3.3. Let $f, g, h$ and $k$ be mappings from a metric space $(\mathcal{X}, d)$ into itself satisfying inequality

$$
\begin{aligned}
& \left(\int_{0}^{d(f x, g y)} \omega(t) \mathrm{d} t\right)^{p} \\
\leq & F\left[a\left(\int_{0}^{d(h x, k y)} \omega(t) \mathrm{d} t\right)^{p}+(1-a) \max \left\{\int_{0}^{d(f x, h x)} \omega(t) \mathrm{d} t\right.\right. \\
& \int_{0}^{d(g y, k y)} \omega(t) d t,\left(\int_{0}^{d(f x, h x)} \omega(t) \mathrm{d} t\right)^{\frac{1}{2}}\left(\int_{0}^{d(f x, k y)} \omega(t) \mathrm{d} t\right)^{\frac{1}{2}}, \\
& \left.\left(\int_{0}^{d(h x, g y)} \omega(t) \mathrm{d} t\right)^{\frac{1}{2}}\left(\int_{0}^{d(f x, k y)} \omega(t) \mathrm{d} t\right)^{\frac{1}{2}}\right\}
\end{aligned}
$$

for all $x, y$ in $\mathcal{X}$, where $0<a<1, p$ is an integer such that $p \geq 1, F \in \mathcal{F}$ and $\omega \in \Omega$. If $h$ and $k$ are continuous and the pairs $(f, h)$ and $(g, k)$ are subcompatible, then, $f, g, h$ and $k$ have a unique common fixed point. 
Proof. Since the pair $(f, h)$ as well as $(g, k)$ is subcompatible, then, there are two sequenses $\left\{x_{n}\right\}$ and $\left\{y_{n}\right\}$ in $\mathcal{X}$ such that $\lim _{n \rightarrow \infty} h x_{n}=\lim _{n \rightarrow \infty} f x_{n}=t$ for some $t \in \mathcal{X}$ and $\lim _{n \rightarrow \infty} d\left(f h x_{n}, h f x_{n}\right)=0 ; \lim _{n \rightarrow \infty} g y_{n}=\lim _{n \rightarrow \infty} k y_{n}=z$ for some $z \in \mathcal{X}$ and $\lim _{n \rightarrow \infty} d\left(g k y_{n}, k g y_{n}\right)=0$.

First, we prove that $z=t$. If $t \neq z$, using inequality (2) we get

$$
\begin{aligned}
& \left(\int_{0}^{d\left(f x_{n}, g y_{n}\right)} \omega(t) \mathrm{d} t\right)^{p} \\
\leq & F\left[a\left(\int_{0}^{d\left(h x_{n}, k y_{n}\right)} \omega(t) d t\right)^{p}+(1-a) \max \left\{\int_{0}^{d\left(f x_{n}, h x_{n}\right)} \omega(t) \mathrm{d} t,\right.\right. \\
& \int_{0}^{d\left(g y_{n}, k y_{n}\right)} \omega(t) d t,\left(\int_{0}^{d\left(f x_{n}, h x_{n}\right)} \omega(t) \mathrm{d} t\right)^{\frac{1}{2}}\left(\int_{0}^{d\left(f x_{n}, k y_{n}\right)} \omega(t) \mathrm{d} t\right)^{\frac{1}{2}}, \\
& \left.\left.\left(\int_{0}^{d\left(h x_{n}, g y_{n}\right)} \omega(t) \mathrm{d} t\right)^{\frac{1}{2}}\left(\int_{0}^{d\left(f x_{n}, k y_{n}\right)} \omega(t) \mathrm{d} t\right)^{\frac{1}{2}}\right\}\right]
\end{aligned}
$$

Letting $n \rightarrow \infty$, we obtain

$$
\begin{aligned}
& \left(\int_{0}^{d(t, z)} \omega(t) \mathrm{d} t\right)^{p} \\
\leq & F\left[a\left(\int_{0}^{d(t, z)} \omega(t) \mathrm{d} t\right)^{p}+(1-a)\left(\int_{0}^{d(t, z)} \omega(t) \mathrm{d} t\right)^{p}\right] \\
= & F\left[\left(\int_{0}^{d(t, z)} \omega(t) \mathrm{d} t\right)^{p}\right]<\left(\int_{0}^{d(t, z)} \omega(t) \mathrm{d} t\right)^{p},
\end{aligned}
$$

which is a contradiction, then $\int_{0}^{d(t, z)} \omega(t) \mathrm{d} t=0$, hence $z=t$.

Since $h$ is continuous, then we have $h^{2} x_{n} \rightarrow h t, h f x_{n} \rightarrow h t$. Also, we have

$$
d\left(f h x_{n}, h t\right) \leq d\left(f h x_{n}, h f x_{n}\right)+d\left(h f x_{n}, h t\right) .
$$

As $f$ and $h$ are subcompatible, letting $n$ tends to infinity in the above inequality, we obtain $\lim _{n \rightarrow \infty} f h x_{n}=h t$. The use of condition (2) gives

$$
\begin{aligned}
& \left(\int_{0}^{d\left(f h x_{n}, g y_{n}\right)} \omega(t) \mathrm{d} t\right)^{p} \\
\leq & F\left[a\left(\int_{0}^{d\left(h^{2} x_{n}, k y_{n}\right)} \omega(t) \mathrm{d} t\right)^{p}+(1-a) \max \left\{\int_{0}^{d\left(f h x_{n}, h^{2} x_{n}\right)} \omega(t) \mathrm{d} t,\right.\right.
\end{aligned}
$$




$$
\begin{aligned}
& \int_{0}^{d\left(g y_{n}, k y_{n}\right)} \omega(t) \mathrm{d} t,\left(\int_{0}^{d\left(f h x_{n}, h^{2} x_{n}\right)} \omega(t) \mathrm{d} t\right)^{\frac{1}{2}}\left(\int_{0}^{d\left(f h x_{n}, k y_{n}\right)} \omega(t) \mathrm{d} t\right)^{\frac{1}{2}} \\
& \left.\left.\left(\int_{0}^{d\left(h^{2} x_{n}, g y_{n}\right)} \omega(t) \mathrm{d} t\right)^{\frac{1}{2}}\left(\int_{0}^{d\left(f h x_{n}, k y_{n}\right)} \omega(t) \mathrm{d} t\right)^{\frac{1}{2}}\right\}\right]
\end{aligned}
$$

We obtain at infinity

$$
\begin{array}{rl} 
& \left(\int_{0}^{d(h t, t)} \omega(t) \mathrm{d} t\right)^{p} \\
\leq F & F\left[\left(\int_{0}^{d(h t, t)} \omega(t) \mathrm{d} t\right)^{p}+(1-a)\left(\int_{0}^{d(h t, t)} \omega(t) \mathrm{d} t\right)^{p}\right] \\
= & F\left[\left(\int_{0}^{d(h t, t)} \omega(t) d t\right)^{p}\right]<\left(\int_{0}^{d(h t, t)} \omega(t) \mathrm{d} t\right)^{p},
\end{array}
$$

which is a contradiction, therefore $h t=t$.

Again by inequality (2) we have

$$
\begin{aligned}
& \left(\int_{0}^{d\left(f t, g y_{n}\right)} \omega(t) \mathrm{d} t\right)^{p} \\
\leq & F\left[a\left(\int_{0}^{d\left(h t, k y_{n}\right)} \omega(t) \mathrm{d} t\right)^{p}+(1-a) \max \left\{\int_{0}^{d(f t, h t)} \omega(t) \mathrm{d} t\right.\right. \\
& \int_{0}^{d\left(g y_{n}, k y_{n}\right)} \omega(t) \mathrm{d} t,\left(\int_{0}^{d(f t, h t)} \omega(t) \mathrm{d} t\right)^{\frac{1}{2}}\left(\int_{0}^{d\left(f t, k y_{n}\right)} \omega(t) \mathrm{d} t\right)^{\frac{1}{2}}, \\
& \left.\left(\int_{0}^{d\left(h t, g y_{n}\right)} \omega(t) \mathrm{d} t\right)^{\frac{1}{2}}\left(\int_{0}^{d\left(f t, k y_{n}\right)} \omega(t) \mathrm{d} t\right)^{\frac{1}{2}}\right]
\end{aligned}
$$

At infinity we obtain

$$
\begin{aligned}
\left(\int_{0}^{d(f t, t)} \omega(t) \mathrm{d} t\right)^{p} & \leq F\left[(1-a)\left(\int_{0}^{d(f t, t)} \omega(t) \mathrm{d} t\right)^{p}\right] \\
& <(1-a)\left(\int_{0}^{d(f t, t)} \omega(t) \mathrm{d} t\right)^{p} \\
& <\left(\int_{0}^{d(f t, t)} \omega(t) \mathrm{d} t\right)^{p}
\end{aligned}
$$

which is a contradiction. Hence $f t=t$. 
Now, since $k$ is continuous, then, we have $k^{2} y_{n} \rightarrow k t$ and $k g y_{n} \rightarrow k t$ and

$$
d\left(g k y_{n}, k t\right) \leq d\left(g k y_{n}, k g y_{n}\right)+d\left(k g y_{n}, k t\right) .
$$

Since the pair $(g, k)$ is subcompatible, we get at infinity $\lim _{n \rightarrow \infty} g k y_{n}=k t$. Using (2) we have

$$
\begin{aligned}
& \left(\int_{0}^{d\left(f t, g k y_{n}\right)} \omega(t) \mathrm{d} t\right)^{p} \\
\leq & F\left[a\left(\int_{0}^{d\left(h t, k^{2} y_{n}\right)} \omega(t) \mathrm{d} t\right)^{p}+(1-a) \max \left\{\int_{0}^{d(f t, h t)} \omega(t) \mathrm{d} t,\right.\right. \\
& \int_{0}^{d\left(g k y_{n}, k^{2} y_{n}\right)} \omega(t) \mathrm{d} t,\left(\int_{0}^{d(f t, h t)} \omega(t) \mathrm{d} t\right)^{\frac{1}{2}}\left(\int_{0}^{d\left(f t, k^{2} y_{n}\right)} \omega(t) \mathrm{d} t\right)^{\frac{1}{2}}, \\
& \left.\left.\left(\int_{0}^{d\left(h t, g k y_{n}\right)} \omega(t) \mathrm{d} t\right)^{\frac{1}{2}}\left(\int_{0}^{d\left(f t, k^{2} y_{n}\right)} \omega(t) \mathrm{d} t\right)^{\frac{1}{2}}\right\}^{p}\right]
\end{aligned}
$$

We get at infinity

$$
\begin{aligned}
& \left(\int_{0}^{d(t, k t)} \omega(t) \mathrm{d} t\right)^{p} \\
\leq & F\left[a\left(\int_{0}^{d(t, k t)} \omega(t) \mathrm{d} t\right)^{p}+(1-a)\left(\int_{0}^{d(t, k t)} \omega(t) \mathrm{d} t\right)^{p}\right] \\
= & F\left[\left(\int_{0}^{d(t, k t)} \omega(t) \mathrm{d} t\right)^{p}\right]<\left(\int_{0}^{d(t, k t)} \omega(t) \mathrm{d} t\right)^{p} .
\end{aligned}
$$

This contradiction implies that $k t=t$.

Suppose that $g t \neq t$, the use of inequality (2) gives

$$
\begin{aligned}
& \left(\int_{0}^{d(f t, g t)} \omega(t) \mathrm{d} t\right)^{p} \\
\leq & F\left[a\left(\int_{0}^{d(h t, k t)} \omega(t) \mathrm{d} t\right)^{p}+(1-a) \max \left\{\int_{0}^{d(f t, h t)} \omega(t) \mathrm{d} t,\right.\right. \\
& \int_{0}^{d(g t, k t)} \omega(t) \mathrm{d} t,\left(\int_{0}^{d(f t, h t)} \omega(t) \mathrm{d} t\right)^{\frac{1}{2}}\left(\int_{0}^{d(f t, k t)} \omega(t) \mathrm{d} t\right)^{\frac{1}{2}}, \\
& \left.\left.\left(\int_{0}^{d(h t, g t)} \omega(t) \mathrm{d} t\right)^{\frac{1}{2}}\left(\int_{0}^{d(f t, k t)} \omega(t) \mathrm{d} t\right)^{\frac{1}{2}}\right\}\right]
\end{aligned}
$$


i.e.,

$$
\begin{aligned}
\left(\int_{0}^{d(t, g t)} \omega(t) \mathrm{d} t\right)^{p} & \leq F\left[(1-a)\left(\int_{0}^{d(t, g t)} \omega(t) \mathrm{d} t\right)^{p}\right] \\
& <(1-a)\left(\int_{0}^{d(t, g t)} \omega(t) \mathrm{d} t\right)^{p} \\
& <\left(\int_{0}^{d(t, g t)} \omega(t) \mathrm{d} t\right)^{p},
\end{aligned}
$$

which is a contradiction. Hence $g t=t$. Therefore $t=z$ is a common fixed point of both $f, g, h$ and $k$.

Suppose that $f, g, h$ and $k$ have another common fixed point $z \neq t$. Then, by inequality (2) we get

$$
\begin{aligned}
& \left(\int_{0}^{d(f t, g z)} \omega(t) \mathrm{d} t\right)^{p} \\
\leq & F\left[a\left(\int_{0}^{d(h t, k z)} \omega(t) \mathrm{d} t\right)^{p}+(1-a) \max \left\{\int_{0}^{d(f t, h t)} \omega(t) \mathrm{d} t\right.\right. \\
& \int_{0}^{d(g z, k z)} \omega(t) \mathrm{d} t,\left(\int_{0}^{d(f t, h t)} \omega(t) \mathrm{d} t\right)^{\frac{1}{2}}\left(\int_{0}^{d(f t, k z)} \omega(t) \mathrm{d} t\right)^{\frac{1}{2}}, \\
& \left.\left.\left(\int_{0}^{d(h t, g z)} \omega(t) \mathrm{d} t\right)^{\frac{1}{2}}\left(\int_{0}^{d(f t, k z)} \omega(t) \mathrm{d} t\right)^{\frac{1}{2}}\right\}^{p}\right]
\end{aligned}
$$

that is

$$
\begin{aligned}
\left(\int_{0}^{d(t, z)} \omega(t) \mathrm{d} t\right)^{p} & \leq F\left[\left(\int_{0}^{d(t, z)} \omega(t) \mathrm{d} t\right)^{p}\right] \\
& <\left(\int_{0}^{d(t, z)} \omega(t) \mathrm{d} t\right)^{p} .
\end{aligned}
$$

This contradiction implies that $z=t$.

If $f=g$ and $h=k$ in Theorem 3.3, we get the next result:

Corollary 3.3. Let $f$ and $h$ be two self-mappings of a metric space $(\mathcal{X}, d)$ such that

$$
\begin{aligned}
& \left(\int_{0}^{d(f x, f y)} \omega(t) \mathrm{d} t\right)^{p} \\
\leq & F\left[a\left(\int_{0}^{d(h x, h y)} \omega(t) \mathrm{d} t\right)^{p}+(1-a) \max \left\{\int_{0}^{d(f x, h x)} \omega(t) \mathrm{d} t,\right.\right.
\end{aligned}
$$




$$
\begin{aligned}
& \int_{0}^{d(f y, h y)} \omega(t) \mathrm{d} t,\left(\int_{0}^{d(f x, h x)} \omega(t) \mathrm{d} t\right)^{\frac{1}{2}}\left(\int_{0}^{d(f x, h y)} \omega(t) \mathrm{d} t\right)^{\frac{1}{2}}, \\
& \left.\left(\int_{0}^{d(h x, f y)} \omega(t) \mathrm{d} t\right)^{\frac{1}{2}}\left(\int_{0}^{d(f x, h y)} \omega(t) \mathrm{d} t\right)^{\frac{1}{2}}\right],
\end{aligned}
$$

for all $x, y$ in $\mathcal{X}$, where $0<a<1, p$ is an integer such that $p \geq 1, F \in \mathcal{F}$ and $\omega \in \Omega$. If $h$ is continuous and the pair $(f, h)$ is subcompatible, then, $f$ and $h$ have a unique common fixed point.

If we let in Theorem $3.3 h=k$, then we get the following corollary:

Corollary 3.4. Let $f, g$ and $h$ be three self-mappings of a metric space $(\mathcal{X}, d)$ such that

$$
\begin{aligned}
& \left(\int_{0}^{d(f x, g y)} \omega(t) \mathrm{d} t\right)^{p} \\
\leq & F\left[a\left(\int_{0}^{d(h x, h y)} \omega(t) \mathrm{d} t\right)^{p}+(1-a) \max \left\{\int_{0}^{d(f x, h x)} \omega(t) \mathrm{d} t\right.\right. \\
& \int_{0}^{d(g y, h y)} \omega(t) \mathrm{d} t,\left(\int_{0}^{d(f x, h x)} \omega(t) \mathrm{d} t\right)^{\frac{1}{2}}\left(\int_{0}^{d(f x, h y)} \omega(t) \mathrm{d} t\right)^{\frac{1}{2}}, \\
& \left.\left(\int_{0}^{d(h x, g y)} \omega(t) \mathrm{d} t\right)^{\frac{1}{2}}\left(\int_{0}^{d(f x, h y)} \omega(t) \mathrm{d} t\right)^{\frac{1}{2}}\right\}
\end{aligned}
$$

for all $x, y$ in $\mathcal{X}$, where $0<a<1, p$ is an integer such that $p \geq 1, F \in \mathcal{F}$ and $\omega \in \Omega$. If $h$ is continuous and the pairs $(f, h)$ and $(g, h)$ are subcompatible, then, $f, g$ and $h$ have a unique common fixed point.

The next result is a generalization of Theorem 3.3.

Theorem 3.4. Let $h, k$ and $\left\{f_{n}\right\}_{n \in \mathbb{N}^{*}}$ be self-mappings of a metric space $(\mathcal{X}, d)$ satisfying the inequality

$$
\begin{aligned}
& \left(\int_{0}^{d\left(f_{n} x, f_{n+1} y\right)} \omega(t) \mathrm{d} t\right)^{p} \\
\leq & F\left[a\left(\int_{0}^{d(h x, k y)} \omega(t) \mathrm{d} t\right)^{p}+(1-a) \max \left\{\int_{0}^{d\left(f_{n} x, h x\right)} \omega(t) \mathrm{d} t,\right.\right. \\
& \int_{0}^{d\left(f_{n+1} y, k y\right)} \omega(t) \mathrm{d} t,\left(\int_{0}^{d\left(f_{n} x, h x\right)} \omega(t) \mathrm{d} t\right)^{\frac{1}{2}}\left(\int_{0}^{d\left(f_{n} x, k y\right)} \omega(t) \mathrm{d} t\right)^{\frac{1}{2}},
\end{aligned}
$$




$$
\left.\left.\left(\int_{0}^{d\left(h x, f_{n+1} y\right)} \omega(t) \mathrm{d} t\right)^{\frac{1}{2}}\left(\int_{0}^{d\left(f_{n} x, k y\right)} \omega(t) \mathrm{d} t\right)^{\frac{1}{2}}\right\}^{p}\right]
$$

for all $x, y$ in $\mathcal{X}$, where $0<a<1, p$ is an integer such that $p \geq 1, F \in \mathcal{F}$ and $\omega \in \Omega$. If $h$ and $k$ are continuous and the pairs $\left(f_{n}, h\right)$ and $\left(f_{n+1}, k\right)$ are subcompatible, then $h, k$ and $\left\{f_{n}\right\}_{n \in \mathbb{N}^{*}}$ have a unique common fixed point.

We end our work by establishing another result which improves, extends and generalizes especially the result of [5].

Theorem 3.5. Let $(\mathcal{X}, d)$ be a metric space, $f, g, h$ and $k$ be mappings from $\mathcal{X}$ into itself and $\boldsymbol{\digamma}$ be an upper semi-continuous function of $[0, \infty)$ into itself such that $\digamma(t)=0$ if and only if $t=0$ and satisfying inequality

$$
\begin{aligned}
& \int_{0}^{\digamma(d(f x, g y))} \omega(t) \mathrm{d} t \\
\leq & a(d(h x, k y)) \int_{0}^{\digamma(d(h x, k y))} \omega(t) \mathrm{d} t \\
+ & b(d(h x, k y)) \int_{0}^{\digamma(d(h x, f x))+\digamma(d(k y, g y))} \omega(t) \mathrm{d} t \\
+ & c(d(h x, k y)) \int_{0}^{\min \{\digamma(d(h x, g y)), \digamma(d(k y, f x))\}} \omega(t) \mathrm{d} t,
\end{aligned}
$$

for all $x, y$ in $\mathcal{X}$, where $\omega \in \Omega$ and $a, b, c:[0, \infty) \rightarrow[0,1)$ are upper semicontinuous and satisfying the condition

$$
a(t)+c(t)<1, \quad t>0 .
$$

If the pairs $(f, h)$ and $(g, k)$ are subcompatible and $h$ and $k$ are continuous, then, $f, g, h$ and $k$ have a unique common fixed point.

Proof. Since the pairs $(f, h)$ and $(g, k)$ are subcompatible, then, there exist two sequences $\left\{x_{n}\right\}$ and $\left\{y_{n}\right\}$ in $\mathcal{X}$ such that $\lim _{n \rightarrow \infty} f x_{n}=\lim _{n \rightarrow \infty} h x_{n}=t$ for some $t \in \mathcal{X}$ and $\lim _{n \rightarrow \infty} d\left(f h x_{n}, h f x_{n}\right)=0 ; \lim _{n \rightarrow \infty} g y_{n}=\lim _{n \rightarrow \infty} k y_{n}=z$ for some $z \in \mathcal{X}$ and $\lim _{n \rightarrow \infty} d\left(g k y_{n}, k g y_{n}\right)=0$.

First, we prove that $z=t$. Suppose that $\digamma(d(t, z))>0$, using inequality (3) we get

$$
\begin{aligned}
\int_{0}^{\digamma\left(d\left(f x_{n}, g y_{n}\right)\right)} & \omega(t) \mathrm{d} t \leq a\left(d\left(h x_{n}, k y_{n}\right)\right) \int_{0}^{\digamma\left(d\left(h x_{n}, k y_{n}\right)\right)} \omega(t) \mathrm{d} t \\
& +b\left(d\left(h x_{n}, k y_{n}\right)\right) \int_{0}^{\digamma\left(d\left(h x_{n}, f x_{n}\right)\right)+\digamma\left(d\left(k y_{n}, g y_{n}\right)\right)} \omega(t) \mathrm{d} t \\
& +c\left(d\left(h x_{n}, k y_{n}\right)\right) \int_{0}^{\min \left\{\digamma\left(d\left(h x_{n}, g y_{n}\right)\right), \digamma\left(d\left(k y_{n}, f x_{n}\right)\right)\right\}} \omega(t) \mathrm{d} t .
\end{aligned}
$$


Taking the limit as $n \rightarrow \infty$, we obtain

$$
\begin{aligned}
\int_{0}^{\digamma(d(t, z))} \omega(t) \mathrm{d} t & \leq[a(d(t, z))+c(d(t, z))] \int_{0}^{\digamma(d(t, z))} \omega(t) \mathrm{d} t \\
& <\int_{0}^{\digamma(d(t, z))} \omega(t) \mathrm{d} t,
\end{aligned}
$$

which is a contradiction. Hence $\digamma(d(t, z))=0$ which implies that $d(t, z)=0$, thus $t=z$.

Since $h$ is continuous, then, we have $h^{2} x_{n} \rightarrow h t, h f x_{n} \rightarrow h t$. Also, we have

$$
d\left(f h x_{n}, h t\right) \leq d\left(f h x_{n}, h f x_{n}\right)+d\left(h f x_{n}, h t\right) .
$$

As $f$ and $h$ are subcompatible, letting $n$ tends to infinity in the above inequality, we obtain $\lim _{n \rightarrow \infty} f h x_{n}=h t$. If $\digamma(d(h t, t))>0$, the use of condition (3) gives

$$
\begin{aligned}
\int_{0}^{\digamma\left(d\left(f h x_{n}, g y_{n}\right)\right)} \omega(t) \mathrm{d} t \leq a\left(d\left(h^{2} x_{n}, k y_{n}\right)\right) \int_{0}^{\digamma\left(d\left(h^{2} x_{n}, k y_{n}\right)\right)} \omega(t) \mathrm{d} t \\
+b\left(d\left(h^{2} x_{n}, k y_{n}\right)\right) \int_{0}^{\digamma\left(d\left(h^{2} x_{n}, f h x_{n}\right)\right)+\digamma\left(d\left(k y_{n}, g y_{n}\right)\right)} \omega(t) \mathrm{d} t \\
+c\left(d\left(h^{2} x_{n}, k y_{n}\right)\right) \int_{0}^{\min \left\{\digamma\left(d\left(h^{2} x_{n}, g y_{n}\right)\right), \digamma\left(d\left(k y_{n}, f h x_{n}\right)\right)\right\}} \omega(t) \mathrm{d} t .
\end{aligned}
$$

Letting $n \rightarrow \infty$ we obtain

$$
\begin{aligned}
\int_{0}^{\digamma(d(h t, t))} \omega(t) \mathrm{d} t & \leq[a(d(h t, t))+c(d(h t, t))] \int_{0}^{\digamma(d(h t, t))} \omega(t) \mathrm{d} t \\
& <\int_{0}^{\digamma(d(h t, t))} \omega(t) \mathrm{d} t .
\end{aligned}
$$

This contradiction implies that $\digamma(d(h t, t))=0$ and hence $h t=t$.

Suppose that $\digamma(d(f t, t))>0$, using condition (3) we get

$$
\begin{aligned}
\int_{0}^{\digamma\left(d\left(f t, g y_{n}\right)\right)} \omega(t) \mathrm{d} t & \leq a\left(d\left(h t, k y_{n}\right)\right) \int_{0}^{\digamma\left(d\left(h t, k y_{n}\right)\right)} \omega(t) \mathrm{d} t \\
& +b\left(d\left(h t, k y_{n}\right)\right) \int_{0}^{\digamma(d(h t, f t))+\digamma\left(d\left(k y_{n}, g y_{n}\right)\right)} \omega(t) \mathrm{d} t \\
& +c\left(d\left(h t, k y_{n}\right)\right) \int_{0}^{\min \left\{\digamma\left(d\left(h t, g y_{n}\right)\right), \digamma\left(d\left(k y_{n}, f t\right)\right)\right\}} \omega(t) \mathrm{d} t .
\end{aligned}
$$

We obtain at infinity

$$
\int_{0}^{\digamma(d(f t, t))} \omega(t) \mathrm{d} t \leq b(0) \int_{0}^{\digamma(d(t, f t))} \omega(t) \mathrm{d} t<\int_{0}^{\digamma(d(f t, t))} \omega(t) \mathrm{d} t,
$$

which is a contradiction, hence $\digamma(d(f t, t))=0$ which implies that $f t=t$. 
Now, since $k$ is continuous, then, we have $k^{2} y_{n} \rightarrow k t, k g y_{n} \rightarrow k t$ and

$$
d\left(g k y_{n}, k t\right) \leq d\left(g k y_{n}, k g y_{n}\right)+d\left(k g y_{n}, k t\right) .
$$

Since the pair $(g, k)$ is subcompatible, we get at infinity $\lim _{n \rightarrow \infty} g k y_{n}=k t$. We claim that $k t=t$, if not, then by (3) we have

$$
\begin{aligned}
\int_{0}^{\digamma\left(d\left(f t, g k y_{n}\right)\right)} \omega(t) \mathrm{d} t & \leq a\left(d\left(h t, k^{2} y_{n}\right)\right) \int_{0}^{\digamma\left(d\left(h t, k^{2} y_{n}\right)\right)} \omega(t) \mathrm{d} t \\
+ & b\left(d\left(h t, k^{2} y_{n}\right)\right) \int_{0}^{\digamma(d(h t, f t))+\digamma\left(d\left(k^{2} y_{n}, g k y_{n}\right)\right)} \omega(t) \mathrm{d} t \\
& +c\left(d\left(h t, k^{2} y_{n}\right)\right) \int_{0}^{\min \left\{\digamma\left(d\left(h t, g k y_{n}\right)\right), \digamma\left(d\left(k^{2} y_{n}, f t\right)\right)\right\}} \omega(t) \mathrm{d} t .
\end{aligned}
$$

Taking the limit when $n \rightarrow \infty$ we have

$$
\begin{aligned}
\int_{0}^{\digamma(d(t, k t))} \omega(t) \mathrm{d} t & \leq[a(d(t, k t))+c(d(t, k t))] \int_{0}^{\digamma(d(t, k t))} \omega(t) \mathrm{d} t \\
& <\int_{0}^{\digamma(d(t, k t))} \omega(t) \mathrm{d} t \\
\Phi(d(t, k t)) & \leq[a(d(t, k t))+c(d(t, k t))] \Phi(d(t, k t)) \\
& <\Phi(d(t, k t)),
\end{aligned}
$$

which is a contradiction, thus $k t=t$.

Suppose that $\digamma(d(t, g t))>0$, then the use of inequality (3) yields

$$
\begin{aligned}
\int_{0}^{\digamma(d(t, g t))} \omega(t) \mathrm{d} t= & \int_{0}^{\digamma(d(f t, g t))} \omega(t) \mathrm{d} t \\
\leq & a(d(h t, k t)) \int_{0}^{\digamma(d(h t, k t))} \omega(t) \mathrm{d} t \\
& +b(d(h t, k t)) \int_{0}^{\digamma(d(h t, f t))+\digamma(d(k t, g t))} \omega(t) \mathrm{d} t \\
& +c(d(h t, k t)) \int_{0}^{\min \{\digamma(d(h t, g t)), \digamma(d(k t, f t))\}} \omega(t) \mathrm{d} t \\
= & b(0) \int_{0}^{\digamma(d(t, g t))} \omega(t) \mathrm{d} t<\int_{0}^{\digamma(d(t, g t))} \omega(t) \mathrm{d} t,
\end{aligned}
$$

which is a contradiction, thus $\digamma(d(t, g t))=0$ which implies that $d(t, g t)=0$ i.e. $g t=t$.

Now, assume that there exists another common fixed point $z$ of $f, g, h$ and $k$ such that $z \neq t$. By inequality (3) we obtain

$$
\int_{0}^{\digamma(d(t, z))} \omega(t) \mathrm{d} t=\int_{0}^{\digamma(d(f t, g z))} \omega(t) \mathrm{d} t
$$




$$
\begin{aligned}
& \leq \quad a(d(h t, k z)) \int_{0}^{\digamma(d(h t, k z))} \omega(t) \mathrm{d} t \\
& \quad+b(d(h t, k z)) \int_{0}^{\digamma(d(h t, f t))+\digamma(d(k z, g z))} \omega(t) \mathrm{d} t \\
& \quad+c(d(h t, k z)) \int_{0}^{\min \{\digamma(d(h t, g z)), \digamma(d(k z, f t))\}} \omega(t) \mathrm{d} t \\
& =[a(d(t, z))+c(d(t, z))] \int_{0}^{\digamma(d(t, z))} \omega(t) \mathrm{d} t \\
& <\int_{0}^{\digamma(d(t, z))} \omega(t) \mathrm{d} t .
\end{aligned}
$$

This contradiction implies that $\digamma(d(t, z))=0 \Leftrightarrow d(t, z)=0$, hence $z=$ $t$.

Remark 3.1. Theorem 3.5 remains valid if we replace inequality (3) by the following one

$$
\begin{aligned}
\int_{0}^{\digamma(d(f x, g y))} \omega(t) \mathrm{d} t \leq & a(d(h x, k y)) \int_{0}^{\digamma(d(h x, k y))} \omega(t) \mathrm{d} t \\
& +b(d(h x, k y)) \int_{0}^{\frac{\digamma(d(h x, f x))+\digamma(d(k y, g y))}{2}} \omega(t) \mathrm{d} t \\
& +c(d(h x, k y)) \int_{0}^{\frac{\digamma(d(h x, g y))+\digamma(d(k y, f x))}{2}} \omega(t) \mathrm{d} t .
\end{aligned}
$$

Corollary 3.5. Let $f$ and $h$ be self-mappings of a metric space $(\mathcal{X}, d)$. Assume that $h$ is continuous, the pair $(f, h)$ is subcompatible and satisfies the inequality

$$
\begin{aligned}
\int_{0}^{\digamma(d(f x, f y))} \omega(t) \mathrm{d} t \leq & a(d(h x, h y)) \int_{0}^{\digamma(d(h x, h y))} \omega(t) \mathrm{d} t \\
& +b(d(h x, h y)) \int_{0}^{\digamma(d(h x, f x))+\digamma(d(h y, f y))} \omega(t) \mathrm{d} t \\
& +c(d(h x, h y)) \int_{0}^{\min \{\digamma(d(h x, f y)), \digamma(d(h y, f x))\}} \omega(t) \mathrm{d} t,
\end{aligned}
$$

for all $x, y$ in $\mathcal{X}$, where $\digamma, \omega, a, b$ and $c$ are as in Theorem 3.5. Then, $f$ and $h$ have a unique common fixed point.

Corollary 3.6. Let $f, g, h: \mathcal{X} \rightarrow \mathcal{X}$ be mappings satisfying the following inequality

$$
\int_{0}^{\digamma(d(f x, g y))} \omega(t) \mathrm{d} t \leq a(d(h x, h y)) \int_{0}^{\digamma(d(h x, h y))} \omega(t) \mathrm{d} t
$$




$$
\begin{aligned}
& +b(d(h x, h y)) \int_{0}^{\digamma(d(h x, f x))+\digamma(d(h y, g y))} \omega(t) \mathrm{d} t \\
& +c(d(h x, h y)) \int_{0}^{\min \{\digamma(d(h x, g y)), \digamma(d(h y, f x))\}} \omega(t) \mathrm{d} t
\end{aligned}
$$

for all $x, y$ in $\mathcal{X}$, where $\digamma, \omega, a, b$ and $c$ are as in Theorem 3.5. If $h$ is continuous and the pairs $(f, h)$ and $(g, h)$ are subcompatible, then, $f, g$ and $h$ have a unique common fixed point.

Now, we give a generalization of Theorem 3.5.

Theorem 3.6. Let $(\mathcal{X}, d)$ be a metric space, $h, k,\left\{f_{n}\right\}_{n \in \mathbb{N}^{*}}$ be mappings from $\mathcal{X}$ into itself and $\digamma$ be an upper semi-continuous function of $[0, \infty)$ into itself such that $\digamma(t)=0$ if and only if $t=0$ and satisfying the inequality

$$
\begin{aligned}
\int_{0}^{\digamma\left(d\left(f_{n} x, f_{n+1} y\right)\right)} \omega(t) \mathrm{d} t \leq a(d(h x, k y)) \int_{0}^{\digamma(d(h x, k y))} \omega(t) \mathrm{d} t \\
+b(d(h x, k y)) \int_{0}^{\digamma\left(d\left(h x, f_{n} x\right)\right)+\digamma\left(d\left(k y, f_{n+1} y\right)\right)} \omega(t) \mathrm{d} t \\
+c(d(h x, k y)) \int_{0}^{\min \left\{\digamma\left(d\left(h x, f_{n+1} y\right)\right), \digamma\left(d\left(k y, f_{n} x\right)\right)\right\}} \omega(t) \mathrm{d} t
\end{aligned}
$$

for all $x, y$ in $\mathcal{X}$, where $\omega \in \Omega, a, b, c:[0, \infty) \rightarrow[0,1)$ are upper semicontinuous and satisfying the condition

$$
a(t)+c(t)<1, \quad t>0 .
$$

If the pairs $\left(f_{n}, h\right)$ and $\left(f_{n+1}, k\right)$ are subcompatible and $h$ and $k$ are continuous, then $h, k$ and $\left\{f_{n}\right\}_{n \in \mathbb{N}^{*}}$ have a unique common fixed point.

\section{REFERENCES}

[1] A. Djoudi, A. Aliouche, Common fixed point theorems of Greguš type for weakly compatible mappings satisfying contractive conditions of integral type, J. Math. Anal. Appl., 329 (1) (2007), 31-45.

[2] D. Gopal, R. K. Bisht, Metrical common fixed points and noncommuting type mappings, Chapman and Hall/CRC Press, (2017), 29-67.

[3] G. Jungck, Compatible mappings and common fixed points, Internat. J. Math. Math. Sci., 9 (4) (1986), 771-779.

[4] G. Jungck, Common fixed points for noncontinuous nonself maps on nonmetric spaces, Far East J. Math. Sci., 4 (2) (1996), 199-215.

[5] A. Mbarki, A common fixed point theorem for near-contractive mappings, Southwest J. Pure Appl. Math., (1) (2002), 120-125.

[6] H. K. Pathak, R. Tiwari, M. S. Khan, A common fixed point theorem satisfying integral type implicit relations, Appl. Math. E-Notes, 7 (2007), 222-228. 
[7] S. Sessa, On a weak commutativity condition of mappings in fixed point considerations, Publ. Inst. Math. (Beograd) (N.S.), 32 (46) (1982), 149-153.

HAKIMA BouHADJERA

LABORATORY OF Applied Mathematics Badji Mokhtar-Annaba University P.O. Box 12, 23000 Annaba Algeria

E-mail address: b_hakima2000@yahoo.fr 